\title{
Targeting of the kynurenic acid across the blood-brain barrier by core-shell nanoparticles
}

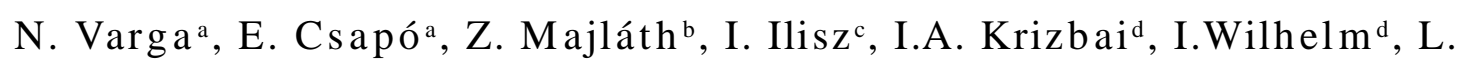
Knapp ${ }^{e}$, J. Toldie,f, L. Vécsei ${ }^{b, e}$, I. Dékány ${ }^{a, *}$

a MTA-SZTE Supramolecular and Nanostructured Materials Research Group, Department of Medical Che mistry, Faculty of Medicine, University of Szeged, H-6720 Dóm tér 8., Szeged, Hungary

${ }^{b}$ Department of Neurology, University of Szeged, H-6725 Semmelweis u. 6, Szeged, Hungaryc Department of Inorganic and Analytical Che mistry, University of Szeged, H-6720 Dóm tér 7, Szeged, Hungary

${ }^{d}$ Institute of Biophysics, Biological Research Centre of the Hungarian Academy of Sciences,

H-6726 Temesvári krt. 62, Szeged, Hungary

${ }^{e}$ Department of Physiology, Anatomy and Neuroscience, University of Szeged, H-6726 Közép fasor 52, Szeged, Hungary

${ }^{f}$ MTA-SZTE Neuroscience Research Group, H-6725 Semmelweis u. 6, Szeged, Hungary

* Corresponding author at: MTA-SZTE Supramolecular and Nanostructured Materials Research Group, Department of Medical Chemistry, Faculty of Medicine, University of Szeged E-mail address:i.dekany@chem.u-szeged.hu (I. Dékány)

\section{Abstract}

Core-shell nanoparticles (CSNPs) were developed to get over therapeutic amount of kynurenic acid (KYNA) across the blood-brain barrier (BBB). Bovine serum albumin (BSA) was used as core for encapsulation of KYNA and the BSA/KYNA composite were finally encapsulated by poly(allylamine) hydrochloride (PAH) polymer as shell. In the interest of the optimization of the synthesis the BSA and KYNA interaction was studied by two-dimensional surface plasmon resonance (SPR) technique as well. The average size of $\mathrm{d} \sim 100 \mathrm{~nm}$ was proven by dynamic light scattering (DLS) and transmission electron microscopy (TEM), while the structure of the composites were characterized by fluorescence (FL) and circular dichroism (CD) spectroscopy. The in vitro release properties of KYNA were investigated by a vertical diffusion cell at $25.0^{\circ} \mathrm{C}$ and $37.5^{\circ} \mathrm{C}$ 
and the kinetic of the release were discussed. The penetration capacity of the NPs into the central nervous system (CNS) was tested by an in vitro BBB model. The results demonstrated that the encapsulated KYNA had significantly higher permeability compared to free KYNA molecules. In the neurobiological serial of in vivo experiments the effects of peripherally administered KYNA with CSNPs were studied in comparison with untreated KYNA. These results clearly proved that KYNA in the CSNPs, administrated peripherally is suitable to cross the BBB and to induce electrophysiological effects within the CNS. As the neuroprotective properties of KYNA nowadays are proven, the importance of the results is obvious.

Keywords: kynurenic acid, core-shell nanoparticles, release, release kinetic, BBB model

\section{List of abbreviations}

BSA, bovine serum albumin; BBB, blood-brain barrier; CD, circular dichroism; CNS, central nervous system; CSNPs, core-shell nanoparticles; DLS, dynamic light scattering; FL, fluorescence spectroscopy; HPLC-MS, high performance liquid chromatography-mass spectrometry; IEP, isoelectric point, KYNA, kynurenic acid; L-KYN, L-kynurenine; PAH, poly(allylamine) hydrochloride; PBS, phosphate buffer; RBECs, rat brain endothelial cells; SEPs, somatosensory evoked responses; SPR, surface plasmon resonance; TEM, transmission electron microscopy; Trp, tryptophan; 


\section{Introduction}

Many potential therapeutic agents that are used in biological systems are limited because they can't get through the BBB, thanks to their properties, for example size, hydrophilic character, charge, etc. This barrier can be circumvented by specific transporters, chemical modifications, interactions with other molecules or application of composites (Gabthuler, 2010). The kynurenine pathway is the main route of tryptophan (Trp) metabolism in mammalian brain, which involves several neuroactive compounds. KYNA is a product of the Trp metabolism, it has a neuroprotective and neuroinhibitory properties (Stone, 1993; Marosi et al., 2010; Sas et al., 2007). It exerts its effect mainly by antagonizing ionotropic glutamate receptors, its neuroprotective capacity can be attributed mainly to the inhibition of the N-methyl-D-aspartate (NMDA) receptors. KYNA can also influence the presynaptic glutamate release by antagonizing the $\alpha 7$-nicotinicactylcholine receptors (Carpenedo et al., 2001; Zadori et al., 2009). KYNA might play important roles in modulating neurotransmission in the CNS (Fukushima et al., 2007). Alterations of the kynurenine metabolites have been demonstrated in several neurological disorders such as Huntington's disease, migraine or stroke (Darlington et al., 2007; Vécsei et al., 2013). Elevating the level of the neuroprotective KYNA in the brain has been demonstrated to be of therapeutic value in animal models of Parkinson's disease, Huntington's disease and migraine (Fejes et al., 2011; Silva-Adaya et al., 2011). However, systemic administration of KYNA is not reasonable, because its penetration through the BBB is very poor. In our recent studies, we synthesized a new compound, glucosamine-kynurenic acid (KYNA-NH-GLUC), which in peripheral administration was able to cross the BBB, and to induce reductions in the amplitudes of the evoked responses, similar to those of KYNA administrated intracerebroventricularly (Füvesi et al., 2004). Afterwards, other effective KYNA derivatives were synthesized which were able to cross the $\mathrm{BBB}$ and to induce neuroprotective effects in case of peripheral administration too (Nagy et al., 2011; Fülöp et al., 2012). 
Application of novel, nanotechnology-based carrier systems may promote drug delivery into the brain, which may offer future therapeutic options for several neurological disorders. Different nanocarrier systems have been developed and early in vitro and preclinical studies yielded promising results to achieve drug delivery through the BBB (Wong et al., 2012; Lu et al., 2014). Nowadays, the application of CSNPs has become an area of intense growing interest. The albumin-based NPs are considered to be attractive opportunity as carrier systems because many binding sites are reachable to various drug molecules. The albumins have several specific advantages in nano-scale range, such as biodegradability, biocompatibility and non-toxicity (Elzoghby et al., 2012) so the BSA is widely used for drug delivery, using carriers such as microparticles, nanospheres, NPs and gels (Wang et al., 2008). The secondary structure of the BSA can be examined by CD spectroscopy since it has two negative bands $(208 \mathrm{~nm}, 222 \mathrm{~nm})$ in the far UV region; the first band is characteristic for $\alpha$-helical structure of the protein (Kelly et al., 2005) which can be calculated from the observed ellipticity values (Mandal et al., 2010). To encapsulation of different molecules frequently polymers or polyelectrolytes, such the poly(lactic-co-glycolicacid (PLGA) (Rafati et al., 2012), polyethylene glycol (PEG) (Ashjari et al., 2012), chitosan (Bowman et al., 2006) or the PAH are used. The PAH is a synthetic cationic polyelectrolyte which is water-soluble and biodegradable, so it used in nanotechnology and nanomedical field as well (Zhou et al., 2004; Wytrwal et al., 2014).

In this study, BSA-based CSNPs were developed for KYNA encapsulation. Size and structural information were gained by DLS, TEM and CD methods. In vitro measurements were performed to study the release properties and the release mechanism of the KYNA. An in vitro BBB model was applied to assess the penetration capacity of the encapsulated KYNA compared to free KYNA. In the in vivo neurophysiological studies, the effects of intraperitoneally (i.p.) administered KYNA, its prodrug $L$-kynurenine $(L-K Y N)$, BSA/PAH and BSA/KYNA/PAH were studied. 


\section{Materials and Methods}

\subsection{Materials}

The BSA (fraction V), the KYNA, the PAH with $\mathrm{M}_{\mathrm{w}}$ of $15.000 \mathrm{~g} \mathrm{~mol}^{-1}$, the components of the phosphate buffer (PBS), the sodium phosphate dibasic hexadecahydrate $\left(\mathrm{Na}_{2} \mathrm{HPO}_{4} \times 16 \mathrm{H}_{2} \mathrm{O}\right)$ and the sodium phosphate monobasic monohydrate $\left(\mathrm{NaH}_{2} \mathrm{PO}_{4} \times \mathrm{H}_{2} \mathrm{O}\right)$ were purchased from Sigma Aldrich. The sodium chloride $(\mathrm{NaCl})$ and the sodium hydroxide $(\mathrm{NaOH})$ pastilles was bought from Molar Chemicals. Highly purified water was obtained by deionization and filtration with a Millipore purification apparatus. All solvents and reagents used were of analytical grade and no further purifications were made.

\subsection{Synthesis of CSNPs}

All samples were prepared in PBS buffer $(\mathrm{pH}=7.4)$ at $25.0{ }^{\circ} \mathrm{C}$ with constant ionic strength $(0.9 \% \mathrm{w} / \mathrm{v} \mathrm{NaCl})$. The BSA $(6 \% \mathrm{w} / \mathrm{v})$ was stirred in PBS, after fully dissolving $90 \mathrm{mg}$ KYNA was added to the protein solution (BSA : KYNA molar ratio is $1: 175)$. The BSA/KYNA composite dispersion was stirred for two hours. Then PAH solution $(0.24 \% \mathrm{w} / \mathrm{v}$; PBS, $\mathrm{pH}=7.4)$ was added to the BSA/KYNA composite. The final volume was 3 ml. The products were stored at $-80{ }^{\circ} \mathrm{C}$ because of the stability of the drug and the protein molecules.

\subsection{Materials characterization}

For determination of the charge of the initial materials (BSA, PAH) at pH $=7.4$ the components were titrated with $\mathrm{NaOH}\left(\mathrm{c}_{\mathrm{NaOH}}=1.0 \mathrm{M}\right)$ using particle charge detector (PCD-04 MÜTEK). Moreover, the BSA and the BSA/PAH composite were titrated with KYNA to define the amount of drug molecules in the interest of the charge compensation. The fluorescence spectra were recorded by a Horiba Jobin Yvon Fluoromax-4 spectrofluorometer (excitation at $280 \mathrm{~nm}$ ). The size of the prepared products were determined by DLS method with a Horiba SZ-100 apparatus. Circular Dichroism (CD) spectra were recorded (190-250 nm; 
$25.0{ }^{\circ} \mathrm{C}$; bandwidth: $2 \mathrm{~nm}$; scanning speed: $100 \mathrm{~nm} / \mathrm{min}$ ) using a Jasco J$815 \mathrm{CD}$ spectrometer. During the synthesis for controlling the concentration of the KYNA spectrophotometric measurements were carried out using a UV-1800 (Shimadzu) spectrophotometer (at $333 \mathrm{~nm}$ ). The TEM images were registered by using a FEI Tecnai $G^{2} 20$ X-TWIN microscope (tungsten cathode; $200 \mathrm{kV}$ ). The SPR technique are capable of real-time monitoring of biomolecular interactions on a gold sensor surface without the use of labels (Homola et al. 2008). During SPR measurements, one of the interactants is immobilized from solution onto a solid/liquid interface, and a solution of the other interactant is passed over the functionalized gold surface. During this procedure, the refractive index at the interface undergoes change, this being directly related to the plasmon shift $(\Delta \lambda)$ as well as the amount of the biomolecules $\left(\mathrm{m}^{\mathrm{s}} / \mathrm{ng} \mathrm{cm}^{-2}\right)$ adsorbed on the surface of the biosensor chip. More detailed information of SPR technique (measuring principles, applied equations for calculations) see in Homola et al., 2008 and in our recent publications (Sebők et al., 2013; Csapó et al., 2014; Csapó et al., 2015). SPR measurements were carried out on the two-channel SPR sensor platform developed at the Institute of Photonics and Electronics (Prague). The interaction of KYNA with BSA was studied in the concentration range of

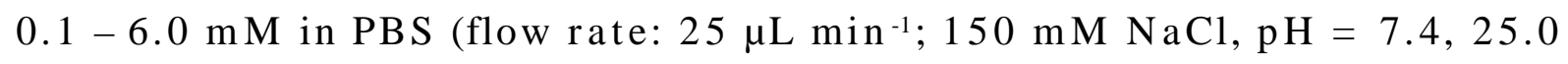
$\left.{ }^{\circ} \mathrm{C}\right)$. Parallel measurements have been performed and the standard deviations of the sorption experiments were $\pm 4.5 \%$. In each steps 800 $800 \mu 1$ protein and KYNA solutions were injected and the sorption time ( $25 \mathrm{~min}$ ) was followed by rinsing procedure using buffer solution. The sensorgrams ( $\Delta \lambda$ vs. time) were analyzed in real-time by a special software package that allows determination of the resonant wavelength in both sensing channels.

\subsection{In vitro drug release experiments}

The in vitro experiments were carried out according to the procedure described earlier (Varga et al., 2014, 2015). Briefly, the nanoparticle dispersion containing KYNA in PBS, $(\mathrm{pH}=7.4)$ was separated by cellulose 
membrane from the physiological solution in a vertical diffusion cell (Franz cell; HANSON CO.). The cell was connected to a UV-1800 spectrophotometer via a peristaltic pump forming a close circulating system. The solution was stirred continuously with a helical magnetic stirrer and the release experiments were carried out at $25.0^{\circ} \mathrm{C}$ and $37.5^{\circ} \mathrm{C}$ as well. Samples were taken every 10 minutes during the first hour, and then only once every hour. Measurements were performed in 500 minutes. The experiment was repeated two times and the standard deviation of the experiments was $\pm 2.5 \%$.

\subsection{Cell culture and permeability assay}

Primary rat brain endothelial cells (RBECs), pericytes and glial cells were isolated as described previously (Wilhelm et al., 2011). The in vitro BBB model was constructed by plating pericytes onto the backside of 12-well Transwell filters (pore size: $0.4 \mu \mathrm{m}$ ) and RBECs onto the upper side. After reaching confluence, the endothelial monolayer was supplied with 550 nM hydrocortisone, $250 \mu \mathrm{M}$ CPT-cAMP (Sigma-Aldrich), and 17.5 $\mu \mathrm{M}$ RO201724 (Roche) and placed into the CellZscope instrument (nanoAnalytics, Muenster, Germany) containing glial-conditioned media. TEER (transendothelial electrical resistance) was followed until reaching plateau of 120-140 Ohm $\times \mathrm{cm}^{2}$. Transwell filters containing endothelial cells and pericytes were removed from the CellZscope instrument. Filters were washed with Ringer-HEPES solution $(\mathrm{pH}=7.4)$. Test substances (KYNA or BSA/KYNA/PAH) were dissolved in Ringer-HEPES and applied in the upper compartment in a final concentration of $20 \mu \mathrm{M}$ KYNA. The lower compartment was loaded with Ringer-HEPES. Samples were taken from the basolateral side after $1 \mathrm{~h}$. The concentrations of the samples were quantitated with an Agilent 1100 HPLC-MS system (Agilent Technologies, Santa Clara, CA, USA). Permeability of the test substances was calculated as described previously (Nagyoszi et al., 2010). Statistical analysis was performed using Student's t-test. 


\subsection{In vivo experiments}

2.6.1. Animals, surgery and electrophysiology

In vivo experiments were performed on adult Wistar rats, weighing $300 \mathrm{~g}$ $(n=30)$. Food and water were available ad libitum. All procedures were approved by the Animal Care Committee of the University and were conducted according to the recommendations of the Declaration of Helsinki and Tokyo. The animals were anesthetized with an intraperitoneal urethane injection (1.3 g / $\mathrm{kg}$ body weight). Body temperature was maintained at $37 \pm 0.5{ }^{\circ} \mathrm{C}$ through the use of a selfregulating heating pad. The somatosensory evoked responses (SEPs) were induced by the electric stimulation of the whiskers with bipolar needle electrodes $(3-3.5 \mathrm{~V} ; 0.2 \mathrm{~ms}, 0.1 \mathrm{~Hz})$. More details of surgery and electrophysiology see in former works (Toldi et al., 1988, 1994).

\subsubsection{Experimental groups and treatment}

KYNA, L-KYN (Sigma-Aldrich, St. Louis, MO, USA), encapsulated KYNA, empty BSA composite and saline $(n=5,5,6,9,5)$ was administered via i.p. injection after $30 \mathrm{~min}$ control period of the recordings (dose: $300 \mathrm{mg} /$ $\mathrm{kg}$; speed: $\left.333 \mu \mathrm{l} \mathrm{min}^{-1} ; \mathrm{pH}=7.4\right)$ with the aid of a microinjection pump (CMA / 100, CMA Microdialysis AB, Kista, Sweden).

\subsubsection{Statistical analysis}

Repeated measurements of SER amplitudes of the control and treated groups were compared separately with the aid of the non-parametric Related-Samples Friedman's Two-Way Analysis of Variance by Ranks.

\section{Results and Discussion}

In order to design different protein-based nanocarrier composite systems containing effective drug molecules the study of the interaction between the protein and the drug agent as well as the determination of the binding capacity of protein towards the drug molecule are crucial. Accordingly, two-dimensional SPR measurements were carried out at 25.0 ${ }^{\circ} \mathrm{C}$. Firstly the studied protein was immobilized onto the gold surface from 
aqueous solution $(\mathrm{c}=0.05 \mathrm{mM})$. The registered $\mathrm{SPR}$ sensorgram is presented in Fig. 1 (A). The gold surface was functionalized with the BSA in two steps. As the registered sensorgram shows $81.5 \%$ of adsorbed BSA remains irreversibly bound at gold surface after rinsing procedure. The binding of BSA on gold surface caused $\Delta \lambda=5.5 \mathrm{~nm}$ plasmon shift (see in Fig. 1 (A) inset) which corresponds to the $\mathrm{m}^{\mathrm{s}}=$ $140.05 \mathrm{ng} \mathrm{cm}^{-2}$ adsorbed amount of BSA (conversion published in Sebők et al., 2013, Homola et al., 2008, Liedberg et al., 1993). ${ }^{1}$ Based on the

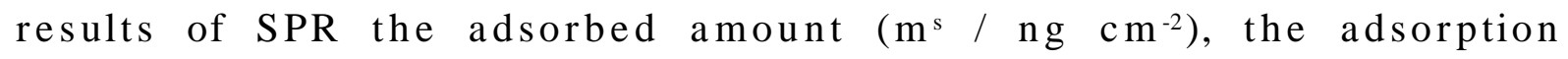
capacity at the maximal concentration $\left(\Gamma_{\mathrm{m}} / \mathrm{nmol} \mathrm{cm}^{-2}\right)$ and the cross sectional area $\left(\mathrm{a}_{\mathrm{m}} / \mathrm{nm}^{2}\right)$ of the studied protein were determined. It was found that the $\Gamma_{\mathrm{m}}=0.002122 \mathrm{nmol} \mathrm{cm}^{-2}$ while the cross sectional area of BSA is $\mathrm{a}_{\mathrm{m}}=78.0 \mathrm{~nm}^{2}$ on gold surface under the applied conditions $\left(\mathrm{a}_{\mathrm{m}}=\right.$ $\left.0.166 \times 1 / \Gamma_{\mathrm{m}}\right)$. In order to determine the binding capacity of KYNA on BSA-covered gold surface the sorption of KYNA on protein-functionalized biosensor chip was investigated at $25.0{ }^{\circ} \mathrm{C}$ in the concentration range of $0.1-6.0 \mathrm{mM}$. The Fig. 1 (B) indicates that increase in concentration of KYNA solutions results in higher wavelength shift from $\sim 0.03 \mathrm{~nm}$ to almost $0.65 \mathrm{~nm}$ (see Fig. 1 (B) secondary y-axes). The sensorgrams also confirm that the interaction between the KYNA drug molecules and this protein is fully reversible because all the adsorbed mass of KYNA reduced to nearly zero after rinsing procedures. This reversible interaction is crucial in order to design potent nanocarrier composite system for (controlled) drug delivery. In the interest of the development of an effective drug delivery systems the interaction between the drug and the carrier should be strong enough to facilitate the transport but also weak enough to release the drug to the target.

Particles charge detector was used to determine the charge of the initial materials as a function of $\mathrm{pH}$. Our results confirm that both the BSA and the PAH are negatively charged at $\mathrm{pH}=7.4$. The isoelectric point (IEP) of BSA and PAH are 4.53 and 5.42, respectively (Fig. 2 (A)).

${ }^{1} \mathrm{~m}^{\mathrm{s}}=\delta \lambda \times\left[\left(\mathrm{S}_{\mathrm{b}} / \mathrm{L}_{\mathrm{p}}\right) \times(\delta \mathrm{n} / \delta \mathrm{c})\right]^{-1} \times 2$, where $\delta \lambda$ is the wavelength(plasmon) shift, $S_{b}$ is the bulk sensitivity, $L_{p}$ is the SP penetration depth, $\delta \mathrm{n} / \delta \mathrm{c}$ is the concentration dependence of the refractive index, Liedberg et al., 1993) 
Hydrogen bonds, hydrophobic and also $\pi-\pi$ interactions can develop between the two materials at physiological pH, unlike electrostatic interactions. Furthermore, the BSA and the BSA/PAH composite were titrated with drug molecules (Fig. 2 (B)) in order to define the a mount of KYNA in the interest of the charge compensation. Based on calculations it was established that $\mathrm{m}=410$ and $\mathrm{m}=425 \mathrm{mg}$ of KYNA is necessary to reach the charge compensation for $1 \mathrm{~g}$ of BSA and BSA/PAH, respectively. During the synthesis we used 1.22- and 1.18-fold excess, respectively.

DLS results (Fig. 3 (A)) clearly confirmed the nanometer size range of the prepared particles. It was found that the size of the negatively charged BSA is about $\mathrm{d}=3.8 \pm 0.3 \mathrm{~nm}$ at $\mathrm{pH}=7.4$. The size of the protein did not change significantly by adding drug molecules (BSA/KYNA composite, $\mathrm{d}=3.6 \pm 0.1 \mathrm{~nm}$ ). When the polyelectrolytes have bound to the BSA in the absence of KYNA, the size increased to only $d=$ $20.5 \pm 1.2 \mathrm{~nm}$. The binding of KYNA to the BSA/PAH composite facilitates the binding of more polyelectrolyte which results in the formation of the CSNPs. The BSA/KYNA/PAH composites have an average size of $d=$ $103.4 \pm 4.8 \mathrm{~nm}$. The registered TEM image shows that the BSA/KYNA/PAH CSNPs have spherical structure and the calculated average diameter $(\mathrm{d}=110 \pm 3.5 \mathrm{~nm})$ is in good agreement with the DLS experiments as well (Fig. 3 (B)).

The fluorescence spectra of the initial materials and the composites are shown in Fig. 4. The BSA has intensive emission peaks at $\lambda=348 \mathrm{~nm}$ using $280 \mathrm{~nm}$ excitation. This high value $(348 \mathrm{~nm})$ indicates that the tryptophan residue (Trp-134) is in contact with bound water molecules (Filenko et al., 2001). As Fig. 4 (A) shows, the continuous addition of KYNA to the protein causes the decrease and also the shift of the emission peak at $\lambda=348 \mathrm{~nm}$ which corresponds to the changes of the polarity around the chromophore molecule. This red shift also indicates that Trp molecules are more exposed to the solvent on average (Klajnert et al., 2003). By adding $10 \mathrm{mM}$ KYNA to the BSA (in this case the BSA : KYNA molar ratio is ca. 1:133), the emission peak of BSA is totally disappeared and only the emission peak at $383 \mathrm{~nm}$ are observed which 
corresponds to the emission of pure KYNA (Fig. 4 (B)). Based on the fluorescence results high KYNA excess (1:175) was used for syntheses. The addition of polyelectrolyte (which does not trigger fluorescence at this excitation wavelength) to the BSA/KYNA nanocomposite caused the decrease in the intensity of the emission peak at $383 \mathrm{~nm}$. This observation probably confirm the encapsulation of BSA/KYNA product by the PAH polymer. It was also observed that, in the absence of KYNA the emission intensity of the BSA is significantly increased by adding PAH to the protein (Fig. 4 (B), curve $e$ ) which support the unfolding of the BSA chains.

CD is a very sensitive method to monitor the conformational change in the protein's secondary structure (Peng et al., 2011). The spectra of the BSA and the CSNPs both in the presence and in the absence of the KYNA are shown in Fig. 5. The spectra of the samples show negative bands located at around $208 \mathrm{~nm}$ and $228 \mathrm{~nm}$. For pure BSA solution a band at $228 \mathrm{~nm}$ is characteristic, the appearance of the other band at 208 $\mathrm{nm}$ is not dominant. The addition of PAH or KYNA to the protein results an intensive reduction and disappearance of this negative band at 228 $\mathrm{nm}$, respectively. These results indicate the binding of KYNA or the polyelectrolyte to the BSA. Most probably the BSA undergoes conformational change; the amount of $\alpha$-helical content is significantly decreased. Based on the equation which was used for calculation (Mandal et al., 2010) the BSA contains $53.3 \%$ of $\alpha$-helical units at $\mathrm{pH}=7.4$. This value is nearly the same for BSA/KYNA system (51.8\%), but the polyelectrolyte causes significant perturbation in the structure of BSA/KYNA composite; it reduces the amount of $\alpha$-helical content to 15.9 $\%$. The PAH (in itself) did not cause significant decrease in the $\alpha$-helical content $(\mathrm{BSA} / \mathrm{PAH}=29.6 \%)$.

In vitro release measurements were carried out over a period of $500 \mathrm{~min}$ (Fig. 6, insets). The KYNA is unstable in solution: at $25.0{ }^{\circ} \mathrm{C}$ (A) and $37.5{ }^{\circ} \mathrm{C}$ (B) the "pure" KYNA decomposes within 2 hours which is delayed by application of composites. The release rate is $55 \%$ after 500 minutes in the case of both BSA/KYNA and the BSA/KYNA/PAH 
composites at $25.0{ }^{\circ} \mathrm{C}$. At higher temperature, the decomposition of the KYNA is faster, significant decrease was observed in the concentration of the KYNA after $500 \mathrm{~min}$. If we study the first hour it can be seen that the amount of KYNA dissolving from the CSNPs $\left(23 \%\right.$ at $\left.25.0{ }^{\circ} \mathrm{C}\right)$ is significantly lower than the amount dissolving from the BSA/KYNA composites $\left(40 \%\right.$ at $\left.25.0{ }^{\circ} \mathrm{C}\right)$. At $37.5{ }^{\circ} \mathrm{C}$ the dissolution is faster: for application of CSNPs $44 \%$ of the KYNA is liberated, while in case of BSA/KYNA composite this value is $53 \%$ after $60 \mathrm{~min}$. Controlled release was achieved by the application of CSNPs, the dissolution of the KYNA molecules were permanent and continuous.

Kinetic models (zero-order rate, first-order rate and Higuchi model) were applied to describe the release mechanism of the KYNA at $25.0{ }^{\circ} \mathrm{C}$ and $37.5{ }^{\circ} \mathrm{C}$ (Varga at el., 2015, Shoaib et al., 2010). The correlation coefficients $\left(\mathrm{R}^{2}\right)$ and the rate constant $\left(\mathrm{k}_{\mathrm{d}}\right)$ values are summarized in Table 1. The Higuchi model, which describes the diffusion-controlled mechanism, fits best for the release of the KYNA at both temperatures. The first-order rate and the Higuchi model are specific for the BSA/KYNA system $\left(\mathrm{R}^{2}>0.98\right)$. It means that the dissolution depends on both the concentration (first-order) and the diffusion (Higuchi). The zero-order rate model characterizes the one-layered composites $\left(\mathrm{R}^{2}>0.98\right)$. This suggests that the drug release rate is independent from the concentration of the dissolved substance. Therefore, constant drug concentration can be maintained by the application of the CSNPs at both temperatures. Compared to the certain rate constants calculated for BSA/KYNA/PAH CSNPs and for pure KYNA one order of magnitude difference is observed (Table 1). This leads to the conclusion that the application of polyelectrolyte shell delay the dissolution of drug molecules implementing the controlled drug release process.

We have assessed the permeability of BSA/KYNA/PAH in comparison to KYNA using an in vitro BBB model. KYNA or BSA/KYNA/PAH was loaded into the upper/blood compartment in a final concentration of 20 $\mu \mathrm{M}$ KYNA and samples were taken from the lower/brain compartment of the model after $1 \mathrm{~h}$ incubation. The permeability coefficient of KYNA was 
$3.71 \times 10^{-6} \mathrm{~cm} / \mathrm{s}\left( \pm 3.64 \times 10^{-7} \mathrm{~cm} / \mathrm{s}\right)$, which was comparable to the permeability of sodium fluorescein $\left(2.96 \times 10^{-6} \mathrm{~cm} / \mathrm{s} \pm 8.78 \times 10^{-7} \mathrm{~cm} /\right.$ s, not shown) measured parallel with the permeability of the test substances. The permeability of KYNA from the BSA/KYNA/PAH composite, however, was significantly (1.9 times) higher: $6.95 \times 10^{-6} \mathrm{~cm} /$ $\mathrm{s}\left( \pm 1.26 \times 10^{-6} \mathrm{~cm} / \mathrm{s}\right)$ (Fig. 7). Our results indicate that KYNA from the BSA/KYNA/PAH composite has a much higher permeability through the BBB than free KYNA. It is well-known that the PAH is a positively charged polymer; therefore, composites encapsulated in PAH are good candidates for adsorptive-mediated transport. This process is triggered by electrostatic interactions between the positively charged substance and the negatively charged glycocalyx of BBB endothelial cells (Gabathuler, 2010; Lu, 2012; Abbott, 2013). Functionalization of the surface of NPs with positively charged biomolecules has been reported several times (Masserini, 2013).

In the animal experiments, in the control group the amplitudes of the SEPs did not changed during the registration period (Fig. 8 (A)). The intraperitoneal injection of KYNA and L-KYN also did not cause significant changes in the amplitudes. (Fig. 8(B)). Inefficacy of KYNA is understandable: KYNA hardly crosses the blood-brain barrier (BBB). The explanation for L-KYN might be in the time course of metabolism (L-KYN $\rightarrow$ KYNA) and/or the low quantity of newly produced KYNA. After administration of BSA/PAH, there was an immediately decrease in the amplitudes for 10-15 min. Later on, it turned to slight facilitation. However, these changes showed no significant difference compare to the control. (Fig. 8 (C)). Experiments in which the animals received BSA/KYNA/PAH treatment, a marked and long-lasting significant decrease in the amplitudes could be observed. Half an hour after the treatment the amplitudes decreased to the $60 \%$ of control. This suppressing effect was kept for 60 min and then the amplitudes started slowly to increase and almost resumed the control level (Fig. 9). Some parts of the neurobiological results are not surprising: since appearance of the excellent paper of Fukui and his co-workers its known that KYNA cross 
hardly the BBB (Fukui et al., 1991). This may explain that i.p. administration of KYNA did not induce any significant changes in the amplitudes of somatosensory evoked responses. Though, L-KYN (pro-drug of KYNA) is actively transported across the BBB by the large neutral amino acid carriers (Fukui et al., 1991) and it is enzymatically converted to KYNA within the CNS, primarily in the astrocytes (Guillemin et al., 2001), the effectiveness of this conversion probably was not enough to produce a marked decrease in the amplitudes of SEPs. Though, one hour after L-KYN administration a slight decrease in the amplitudes started it proved not to be significant. Interestingly, BSA/PAH administration resulted in a transient and slight decrease in the amplitude, which later on turned into slight facilitation. Unfortunately, at this moment we don't know the explanation of these slight changes.

However, in those experiments in which KYNA with CSNPs were administered a long-lasting significant decrease in the amplitudes of SEPs were observed. It suggests that KYNA with CSNPs got through the BBB and was able to diminish the excitatory events in the course of generation of SEPs, within the CNS.

\section{Conclusion}

This study highlighted that a one-pot synthesis technique is applicable to the development of CSNPs for encapsulating KYNA. By application of core-shell structure we were able to overcome the BBB. The reversible binding of drug molecules causes a slight change in the secondary structure of the BSA while a significant perturbation was observed due to the effect of the polyelectrolytes. However, the structure changed: the chains' of the protein unfolded, the BSA/KYNA nanocomposites were enclosed by PAH which is confirmed the results of the in vitro experiments. According to the zero-order kinetic model the calculated rate constant clearly confirm that the drug release process can be wellcontrolled using CSNPs. The efficient application of CSNPs is supported by the neurobiological experiments as well. The KYNA and its pro-drug, the L-KYN did not induce any changes in the amplitudes of somatosensory 
evoked responses. In contrast with this observation, the encapsulated KYNA showed a significant decrease in the evoked signals, so the KYNA successfully penetrated through the BBB by CSNPs. After the KYNA got into the CNS it takes effect and diminish the SEPs value. The results of the in vitro experiments are in good agreement with the in vivo electrophysiological data, both methods confirm that the NPs are able to promote the penetration of KYNA into the brain. Experimental data indicate that KYNA may be neuroprotective and it may be of therapeutic value for several neurological disorders. Nanocarrier systems are suggested to be promising methods for CNS drug delivery, and potential candidates for future drug development.

\section{Acknowledgement}

This research was supported by the European Union and the State of Hungary, co-financed by the European Social Fund in the framework of TÁMOP-4.2.2.A-11/1/KONV-2012-0047, TÁMOP-4.2.6-15/1-2015-0002, TÁMOP-4.2.1.C-14/1/KONV-2015-0013, the Hungarian Research Fund (OTKA PD-100958, K-100807, K 116323, 105077). I.W. by the János Bolyai Research Fellowship of the Hungarian Academy of Sciences (BO/00320/12/8), the project TÁMOP-4.2.2.A-11/1/KONV-2012-0052 by Hungarian Brain Research Programm (NAP, Grant No. KTIA-13-NAP-AIII/19 and KTIA-13-NAP-A-II/17) by EUROHEADPAIN (FP7-Health 2013 Innovation, Grant No 602633) by the MTA-SZTE Neuroscience Research Group of the Hungarian Academy of Sciences and the University of Szeged. 


\section{References}

Abbott, NJ., 2013. Blood-brain barrier structure and function and the challenges for CNS drug delivery. J Inherit Metab Dis. 36, 437-49.

Ashjari, M., Khoee, S., Mahdavian, A.R., Rahmatolahzadeh R., 2012. Selfassembled nanomicelles using PLGA-PEG amphiphilic block copolymer for insulin delivery: a physicochemical investigation and determination of CMC values. J. Mater. Sci.: Mater. Med. 23, 943-953.

Bowman, K., Leong, K.W., 2006. Chitosan nanoparticles for oral drug and gene Delivery. Int. J. Nanomedicine 1, 117-128.

Carpenedo, R., Pittaluga, A., Cozzi, A., Attucci, S., Galli, A., Raiteri, M., Moroni, F., 2001. Presynaptic kynurenate-sensitive receptors inhibit glutamate release. Eur. J. Neurosci., 13, 2141-2147.

Csapó, E., Majláth, Z., Juhász, Á., Roósz, B., Hetényi, A., Tajti, J., Tóth, G.K., Vécsei, L., Dékány, I., 2014. Determination of binding capability and adsorption enthalpy between Human Glutamate Receptor (GluR1) peptide fragments and kynurenic acid by surface plasmon resonance experiments. Coll. Surf. B. 123, 924-929.

Csapó, E., Bogár, F., Juhász, Á., Sebők, D., Szolomájer, J., Majláth, Z., Tóth, G.K., Vécsei, L., Dékány, I., 2015. Determination of binding capability and adsorption enthalpy between Human Glutamate Receptor (GluR1) peptide fragments and kynurenic acid by surface plasmon resonance experiments: Part 2, Interaction of GluR1270-300 with KYNA. Coll. Surf. B. 133, 66-72.

Darlington, L.G., Mackay, G.M., Forrest, C.M., Stoy, N., George C., Stone T.W., 2007. Altered kynurenine metabolism correlates with infarct volume in stroke. Eur. J. Neurosci., 26, 2211-2221.

Elzoghby, A.O., Samy, W.M., Elgindy, N.A., 2012. Albumin-based nanoparticles as potential controlled release drug delivery systems, J. Control. Release 157, 168-182.

Fejes, A., Pardutz, A., Toldi, J., Vécsei, L., 2011. Kynurenine metabolites and migraine: experimental studies and therapeutic perspectives. Curr. Neuropharmacol, 9, 376-87.

Filenko, A., Demchenko, M., Mustafaeva, Z., Osada, Y., Mustafaev M., 2001. Fluorescence study of $\mathrm{Cu}^{2+}$-induced interaction between albumin and anionic polyelectrolytes, Biomacromolecules 2, 270- 277.

Fukui,_S.2 Schwarcz, R., Rapoport,_S.I., Takada,_Y., Smith,_Q.R., 1991. Blood-brain barrier transport of kynurenines: implications for brain synthesis and metabolism. J. Neurochem. 56, 2007-2017.

Fukushima, T., Mitsuhashi, S., Tomiya, M., Iyo, M., Hashimoto, K., Toyo'oka, T., 2007. Determination of kynurenic acid in human serum and its correlation with the concentration of certain amino acids, Clin. Chim. Acta 377, 174-178. 
Fülöp, F., Szatmári, I., Toldi, J., Vécsei, L., 2012. Modifications on the carboxylic function of kynurenic acid. J Neural .Transm.119, 109-114.

Füvesi, J., Somlai,_. , Németh, ․․, Varga, $\underline{\text { H. }}$, Kis, Z.. Farkas, T., Károly, N., Dobszay, M., Penke, Z., Penke,B., Vécsei, L., Toldi, J., 2004. Comparative study on the effects of kynurenic acid and glucosamine-kynurenic acid. Pharmacol. Biochem. Behav. 77, 95- 102.

Gabathuler, R., 2010. Approaches to transport therapeutic drugs across the blood-brain barrier to treat brain diseases. Neurobiol. Dis. 37, $48-57$.

Guillemin, G.J., Kerr, S.J., Smythe, G.A., Smith, D.G., Kapoor, V., Armati, P.J., Croitoru, J., Brew, BJ., 2001. Kynurenine_pathway_metabolism in human astrocytes: a paradox for neuronal protection. J. Neurochem. 78, 842-853.

Homola, J., 2008. Surface Plasmon Resonance Sensors for Detection of

Chemical and Biological Species. Chem. Rev. 108, 462-493.

Kelly, S.M., Jess, T.J., Price, N.C., 2005. How to study proteins by circular dichroism. Biochim. Biophys. Acta 1751, $119-139$.

Klajnert, B., Stanislawska, L., Bryszewska, M., Palecz, B., 2003. Interactions between PAMAM dendrimers and bovine serum albumin. Biochim. Biophys. Acta 1648, 115-126.

Liedberg, B., Lundström, I., Stenberg, E., 1993. Principles of biosensing with an extended coupling matrix and surface plasmon resonance. Sensors and Act. B. 11, 63-72.

Lu, C.T., Zhao, Y.Z., Wong, H.L., Cai, J., Peng, L., Tian, X.Q., 2014. Current approaches to enhance CNS delivery of drugs across the brain barriers. Int. J. Nanomedicine 9, 2241- 2257.

Lu W., 2012. Adsorptive-mediated brain delivery systems. Curr Pharm Biotechnol. 13, 2340-2348.

Mandal, G., Bardhan, M., Ganguly, T., 2010. Interaction of bovine serum albumin and albumin-gold nanoconjugates with L-aspartic acid. A spectroscopic approach, Colloids Surf., B 81, 178-184.

Marosi, M., Nagy, D., Farkas, T., Kis, Z., Rózsa, É., Robotka, H., Fülöp, F., Vécsei, L., Toldi, J., 2010. A novel kynurenic acid analogue: a comparison with kynurenic acid, An in vitro electrophysiological study. J. Neural. Transm. 117, 183-188.

Masserini, M., 2013. Nanoparticles for brain drug delivery. ISRN Biochem. 238428.

Nagy, K., Plangár, I., Tuka, B., Gellért, L., Varga, D., Demeter, I., Farkas, T., Kis, Z., Marosi, M., Zádori, D., Klivényi, P., Fülöp, F., Szatmári, I., Vécsei, L., Toldi, J., 2011. Synthesis and biological effects of some kynurenic acid analogs. Bioorg. Med. Chem. 19, 7590-7596. 
Nagyoszi, P., Wilhelm, I., Farkas, A.E., Fazakas, C., Dung, N.T., Haskó, J., Krizbai, I.A., 2010. Expression and regulation of toll-like receptors in cerebral endothelial cells Neurochem Int. 57, 556-564.

Peng, X., Yao, D., Pan, Y., Yu, Q., Ni, S., Bian, H., Huang, F., Liang, H., 2011. Study on the structural changes of bovine serum albumin with effects on polydatin binding by a multitechnique approach, Spectrochim. Acta Mol. Biomol. Spectros. 81, 209- 214.

Rafati, A., Boussahel, A., Shakesheff, K.M., Shard, A.G., Roberts, C.J., Chen, X., Scurr, D.J., Rigby-Singleton, S., Whiteside, P., Alexander, M.R., Davies, M.C., 2012. Chemical and spatial analysis of protein loaded PLGA microspheres for drug delivery applications. J. Control. Release 162, 321-329.

Sas, K., Robotka, H., Toldi, J., Vécsei L., 2007. Mitochondria, metabolic disturbances, oxidative stress and the kynurenine system, with focus on neurodegenerative disorders. J. Neurol. Sci. 257, 221-239.

Sebők, D; Csapó, E., Preočanin, T., Bohus, G., Kallay, N., Dékány, I., 2013. Adsorption of Ibuprofen and Dopamine on Functionalized Gold Using Surface Plasmon Resonance Spectroscopy at Solid-Liquid Interface. Croat. Chem. Acta 86, 287-295.

Shoaib, M.H., Al Sabah Siddiqi, S., Yousuf, R.I., Zaheer, K., Hanif, M., Rehana, S., Jabeen, S., 2010. Development and Evaluation of Hydrophilic Colloid Matrix of Famotidine Tablets, AAPS Pharm. Sci. Tech. 2, 708-7 18 .

Silva-Adaya, D., Pérez-De La Cruz, V., Villeda-Hernández, J., Carrillo-Mora, P., González- Herrera, I.G., García, E., Colín-Barenque, L., Pedraza-Chaverrí, J., Santamaría, A., 2011. Protective effect of Lkynurenine and probenecid on 6-hydroxydopamine-induced striatal toxicity in rats: implications of modulating kynurenate as a protective strategy. Neurotoxicol Teratol, 33, 303-312.

Stone, T.W., 1993. Neuropharmacology of quinolinic and kynurenic acids. Pharmacol. Rev. 45, 309-379

Toldi, J., Joó, F., Feher, O., Wolff, J.R., 1988. Modified distribution patterns of responses in rat visual cortex induced by monocular enucleation. Neuroscience. 24, 59-66

Toldi, J., Rojik, I., Feher, O., 1994. Neonatal monocular enculeation-induced cross-modal effects observed in the cortex of adult rat. Neuroscience, 62, 105-114.

Varga, N., Benkő, M., Sebők, D., Dékány, I., 2014. BSA/polyelectrolyte core-shell nanoparticles for controlled release of encapsulated ibuprofen. Colloids Surf. B 123, 616-622.

Varga, N., Benkő, M., Sebők, D., Bohus, G., Janovák, L., Dékány, I., 2015. Mesoporous silica core-shell composite functionalized with polyelectrolytes for drug delivery, Micropor. Mesopor. Mat. 213, 134-141. 
Vécsei, L., Szalárdy, L., Fülöp, F., Toldi J., 2013. Kynurenines in the CNS: recent advances and new questions. Nature reviews. Drug discovery, 12, 64-82.

Wang, Y., Wang, X., Luo, G., Dai, Y., 2008. Adsorption of bovine serum albumin (BSA) on to the magnetic chitosan nanoparticles prepared by a microemulsion system, Bioresour. Technol. 99, 3881-3884.

Wilhelm, I., Fazakas, C., Krizbai, I.A., 2011. In vitro models of the bloodbrain barrier. Acta Neurobiol. Exp. (Wars) 71, 113-128.

Wong, H.L., Wu, X.Y., Bendayan, R., 2012. Nanotechnological advances for the delivery of CNS therapeutics. Adv. Drug. Deliv. Rev. 64, 686700 .

Wytrwal, M., Koczurkiewicz, P., Wójcik, K., Michalik, M., Kozik, B., Zylewski, M., Nowakowska, M., Kepczynski, M., 2014. Synthesis of strong polycations with improved biological properties. J. Biomed. Mater. Res. Part A 102A, 721-731.

Zadori, D., Klivényi, P., Vámos, E., Fülöp, F., Toldi, J., Vécsei, L., 2009. Kynurenines in chronic neurodegenerative disorders: future therapeutic strategies. J. Neural. Transm. 116, 1403-1409.

Zhou, Y., Li, Y., 2004. Studies of interaction between poly(allylamine hydrochloride) and double helix DNA by spectral methods. Biophys. Chem. $107,273-281$. 